\title{
Límite de la zona saturada bajo riego por goteo superficial. Mo- delo analítico y empírico.
}

\author{
del Vigo, Á(1). Zubelzu, S.(2), Juana, L. ${ }^{(3)}$.
}

1 Profesor Asociado. Universidad Antonio de Nebrija. Dpto. Ing. Industrial. C/Pirineos 55. 28040 (Madrid). avigo@nebrija.es

2 Profesor Contratado Doctor. ETSI Agronómica, Alimentaria y de Biosistemas de la U. P. de Madrid. Av. Puerta de Hierro 2. 28040 (Madrid). sergio.zubelzu@upm.es

3 Catedrático de Universidad. ETSI Agronómica, Alimentaria y de Biosistemas de la U. P. de Madrid. Av. Puerta de Hierro 2. 28040 (Madrid). luis.juana@upm.es

Resumen: Se ha desarrollado un modelo numérico capaz de calcular la evolución de la zona saturada en superficie bajo condiciones de riego por goteo superficial. Las pruebas realizadas concluyen que el modelo es robusto y eficiente con independencia de las características del suelo. Por otro lado, se ha derivado un modelo analítico simplificado que relaciona el radio de la zona saturada con el caudal aplicado, en base a cuatro parámetros del suelo que son la conductividad hidráulica en saturación, la tensión en el frente y los contenidos de humedad inicial y en saturación del suelo. Además, a partir de simulaciones realizadas con el modelo numérico para suelos del tipo Gardner, Clapp y Hornberger y Van Genuchten-Mualem, se ha obtenido un conjunto de ecuaciones empíricas que relacionan el radio máximo de la zona saturada con el caudal y los parámetros del suelo en cada uno de estos tres esquemas de caracterización. El buen ajuste de estas ecuaciones empíricas con el modelo analítico simplificado justifica su validez. La estimación del radio saturado en superficie junto con el volumen aplicado y la diferencia de contenido de humedad inicial y en saturación del suelo, permite estimar las dimensiones horizontales y verticales del bulbo, lo que es de interés para los proyectos de riego por goteo.

Este trabajo presenta las ecuaciones empíricas obtenidas para el radio máximo de la zona saturada en función del caudal y el tipo de suelo, así como su comparación con el modelo analítico simplificado.

Palabras clave: riego por goteo; tamaño del bulbo; modelo analítico; simulación; ecuaciones empíricas. 


\title{
XXXVIII Congreso Nacional de Riegos CARTAGENA 2021
}

\section{Limit of the saturated zone under surface drip irrigation. Analytical and empirical model}

\author{
del Vigo, Á(1)., Zubelzu, S.(2), Juana, L. ${ }^{(3)}$.
}

1 Profesor Asociado. Universidad Antonio de Nebrija. Dpto. Ing. Industrial. C/Pirineos 55. 28040 (Madrid). avigo@nebrija.es

2 Profesor Contratado Doctor. ETSI Agronómica, Alimentaria y de Biosistemas de la U. P. de Madrid. Av. Puerta de Hierro 2. 28040 (Madrid). sergio.zubelzu@upm.es

3 Catedrático de Universidad. ETSI Agronómica, Alimentaria y de Biosistemas de la U. P. de Madrid. Av. Puerta de Hierro 2. 28040 (Madrid). luis.juana@upm.es

Abstract: A numerical model for the analysis of soil water flow has been developed. Among others, the model is able to predict the evolution of the saturated area on the surface, under superficial drip irrigation. The tests carried out conclude that the model is robust and efficient regardless of the characteristics of the soil. On the other hand, a simplified analytical model has been derived that relates the radius of the saturated zone with the applied flow, based on four parameters of the soil, initial and the saturated soil water contents, the saturated hydraulic conductivity and the wetting front suction head. In addition, the simulations performed for soils of three different type (Gardner, Clapp and Hornberger and Van GenuchtenMualem) achieved a set of empirical equations that relate the maximum radius of the saturated zone with the applied rate flow and the soil parameters for each characterization schemes. The good agreement between these empirical equations and simplified analytical model justifies their validity. The estimation of the maximum saturated radius on the surface as a function of the applied rate flow and soil parameters is of interest for drip irrigation projects.

In this work are presented the empirical equations obtained for the maximum radius of the saturated area under the emitter and their comparison with the simplified analytical model.

Keywords: drip irrigation; bulb size; analytical model; simulation; empirical equations. 


\section{Congreso Nacional de Riegos CARTAGENA 2021}

\section{Introducción}

Los modelos empíricos son de utilidad cuando no existen datos suficientes sobre la naturaleza del suelo como para utilizar modelos analíticos o numéricos de base física, o bien, cuando se requiere una alternativa de sencilla utilización y poca complejidad matemática. La mayor parte de los modelos empíricos existentes relacionan el tamaño del bulbo (radio y profundidad) con el caudal aplicado y el tiempo, teniendo en cuenta algún parámetro del suelo de sencilla obtención experimental como la conductividad hidráulica en saturación, o los contenidos de humedad inicial y en saturación del suelo. En este sentido, algunos modelos de intereses son los modelos empíricos de Schwartzman y Zur [1], Amin y Ekhmaj [2], y otros como por ejemplo [3-6]. La mayor parte de estos modelos están inferidos de observaciones experimentales propias o recogidas de otros autores, y relacionan el radio del frente de avance con el tiempo (o volumen infiltrado).

El radio del frente de avance en superficie que predicen estos modelos es creciente con el tiempo (o volumen aplicado), lo que es una buena aproximación si el tiempo de riego no es muy elevado. Sin embargo, el radio tiende a estabilizarse a un valor constante cuando el tiempo de riego es muy grande o tiende a infinito (régimen estacionario) debido al aumento del área de infiltración, siempre que el caudal permanezca constante. En este contexto, el físico neozelandés R. A. Wooding estableció un modelo analítico a partir de la ecuación de Richards [7] cuya solución en forma de serie infinita tiende a una sencilla expresión de tan solo dos términos que relaciona el tamaño del charco límite (radio máximo, $\boldsymbol{R}_{\mathbf{0}}$ ) con el caudal aplicado $(\boldsymbol{q})$ para condiciones de régimen estacionario:

$$
q=\frac{4 k_{s}}{\alpha} R_{0}+\pi k_{s} R_{0}^{2}
$$

Donde $\boldsymbol{k}_{\boldsymbol{s}}[\mathrm{cm} / \mathrm{min}]$ es la conductividad hidráulica en saturación del suelo y $\boldsymbol{\alpha}\left[\mathrm{cm}^{-1}\right]$ es un parámetro del suelo conocido como constante de capilaridad macroscópica. El primer término de esta ecuación representa el potencial capilar de la propia succión del medio. El segundo término está relacionado con el potencial gravitatorio bajo la fuente. Notar que cuanto más grande es el radio del disco mayor es el efecto gravitatorio frente al de succión.

La limitación del modelo de Wooding se encuentra en que se asume una expresión exponencial para la conductividad hidráulica frente al contenido de humedad asociada al parámetro $\boldsymbol{\alpha}$, conocida como función del tipo Gardner [8], que permite derivar de forma analítica la ecuación (1), pero que no se ajusta a la realidad de la mayor parte de los suelos. Por otro lado, algunos autores como Pullan y Collins [9], Weir [10] o Warrick [11], han reportado un pobre ajuste del modelo de Wooding con respecto a soluciones numéricas propias para casos en los que el radio en régimen estacionario es pequeño.

En este artículo se presenta un grupo de ecuaciones empíricas que relacionan el radio límite de la zona saturada en superficie bajo riego por goteo con el caudal aplicado, la conductividad hidráulica en saturación y la diferencia entre el contenido de humedad inicial y en saturación del suelo. Estas expresiones empíricas se han inferido a partir de resultados de simulación obtenidos con un modelo numérico de elaboración propia [12-15] para suelos caracterizados con funciones del tipo Gardner [8], Clapp y Hornberger [16] y van Genuchten-Mualem [17]. Adicionalmente, se ha derivado desde la ecuación de Darcy y asumiendo hipótesis del tipo Green-Ampt un modelo analítico simplificado [18-19] que describe el caudal infiltrado desde una fuente circular y en superficie de radio constante. 


\section{Materiales y métodos}

\subsection{Modelo de simulación.}

Se ha desarrollado un código en MATLAB [15] que permite estudiar la filtración de agua a través de la ecuación de Richards. El código ha sido ampliamente validado [12,15] por comparación con soluciones analíticas conocidas, otros modelos numéricos como [20] y ensayos experimentales. Por razones de simetría, se utiliza un sistema de coordenadas cilíndricas para la resolución numérica de las ecuaciones, lo que permite reducir el número de variables espaciales y, por tanto, el tiempo de computación. La ecuación de Richards en 3-D, se resuelve en términos del potencial matricial, para cualquier función definida para la conductividad hidráulica $K(\theta)$ [cm/min]; principalmente se han utilizado los modelos de Gardner [8], Clapp y Hornberger [16] y van Genuchten-Mualem [17] para representar esta función. El modelo de simulación utiliza un esquema basado en la técnica de las diferencias finitas para integrar la ecuación de Richards en función del tiempo. Todos los detalles del modelo cómo son su esquema de discretización, el criterio de estabilidad, su validación y otras características pueden encontrarse en la bibliografía [12-15].

\subsection{Modelo analítico simplificado.}

Se ha desarrollado un modelo analítico en tres dimensiones a partir de la ecuación de Darcy y considerando hipótesis del tipo Green-Ampt que describe el volumen y caudal infiltrado desde un emisor superficial semiesférico de radio constante $\left(R_{0}\right)$. A través de argumentos de base física el modelo se ha extendido para el estudio del volumen y caudal infiltrado desde un disco saturado en superficie de radio constante. Se puede consultar todo el desarrollo, las condiciones de contorno y las hipótesis asumidas en la bibliografía [18-19]. El modelo ha sido validado por comparación con resultados de simulación obtenidos con el modelo numérico mencionado en el apartado anterior.

Como resultado del modelo analítico se ha derivado la siguiente ecuación que describe el caudal infiltrado desde el disco $(q)$ cuando el tiempo tiende a infinito, es decir, en régimen estacionario:

$$
\lim _{t \rightarrow \infty} q(t)=\sqrt{2} \pi R_{0}^{2} k_{s} \frac{\tau_{f}}{R_{0}}\left(1+\frac{2 \Delta \theta R_{0}}{\tau_{f}}\right)
$$

Donde $R_{0}$ es el radio del disco [cm] y el resto son parámetros del suelo, siendo $k_{s}[\mathrm{~cm} / \mathrm{min}$ ] la conductividad hidráulica en saturación, $\tau_{f}[\mathrm{~cm}]$ la tensión en el frente de avance y $\Delta \theta=\theta_{s}-\theta_{0}$ es la diferencia entre el contenido de humedad inicial y de saturación del suelo. Se puede expresar la anterior ecuación en magnitudes adimensionales utilizando las siguientes escalas:

$$
c_{g}=1+\frac{2 \Delta \theta R_{0}}{\tau_{f}} ; \quad V_{\text {ref }}=\frac{\sqrt{2}}{3 c_{g}} \pi \Delta \theta R_{0}^{3} ; \quad q_{\text {ref }}=\sqrt{2} \pi R_{0} \tau_{f} k_{s} \cdot c_{g} ; \quad t_{\text {ref }}=\frac{V_{\text {ref }}}{q_{\text {ref }}}
$$




$$
\lim _{t \rightarrow \infty} q^{*}=1
$$

\section{Resultados y discusión}

\subsection{Resultados de simulación y ecuaciones empíricas.}

La Figura 1 representa los resultados obtenidos de las simulaciones realizadas para suelos caracterizados respecto a las funciones de Gardner, Clapp y Hornberger y van Genuchten-Mualem al alcanzar el régimen estacionario para tres regímenes de caudal aplicado distintos $q=2,4,8 L / h$. Se representan los resultados obtenidos para el radio máximo de la zona saturada en superficie $\left(R_{0}\right)$ frente al coeficiente $\sqrt{q / \Delta \theta k_{s}}[\mathrm{~cm}]$, para cada uno de los tres catálogos mencionados:

(a)

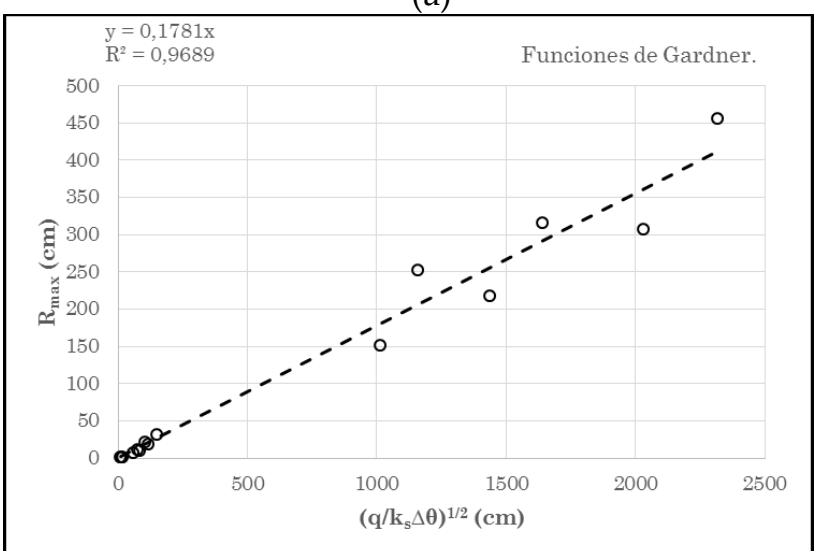

(b)

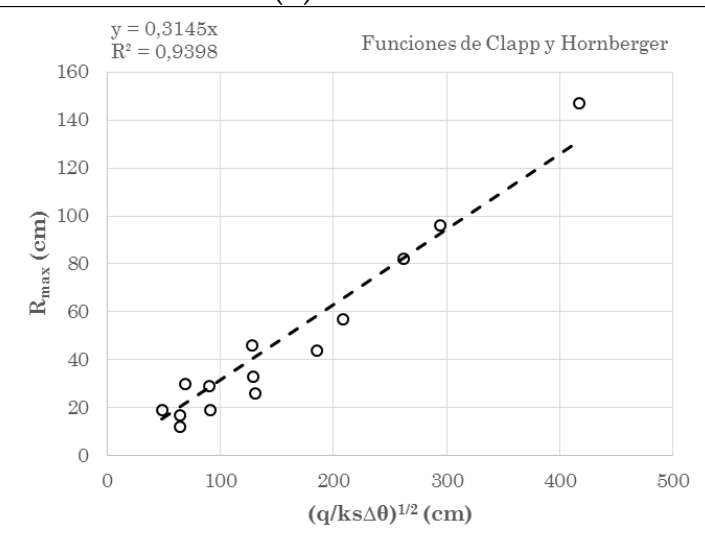

(c)

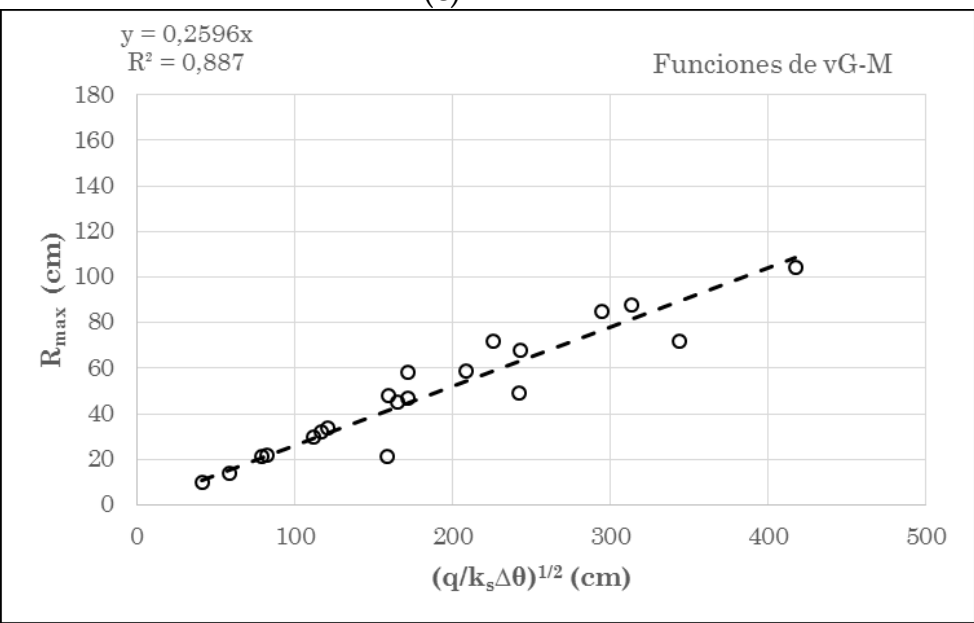

Figura 1. Resultados de las simulaciones realizadas. Radio límite de la zona saturada bajo el gotero una vez alcanzado el régimen estacionario para riego a caudal constante entre $2 \mathrm{~L} / \mathrm{h}$ y $8 \mathrm{~L} / \mathrm{h}$. Suelos de los catálogos de Mualem [21] (a), Clapp y Hornberger [16] (b) y van Genuchten-Mualem [17] (c). 
Se observa una tendencia lineal para los tres tipos de suelo. En la Tabla 1 se muestra el valor estimado de la pendiente [adim] de la recta de ajuste y su error $(\gamma \pm \Delta \gamma)$ para cada una de estas tres gráficas. La Figura 2 presenta dos ejemplos de las simulaciones realizadas para la evolución de la zona saturada en superficie frente al tiempo hasta alcanzar el radio máximo (régimen estacionario). En todas las simulaciones se ha utilizado el criterio de que el régimen estacionario (asintótico) se alcanza cuando el radio máximo permanece al menos el 30\% del tiempo total de simulación con un valor constante.

Tabla 1. Resultados del ajuste del modelo empírico. En la última columna se muestra el coeficiente de correlación lineal obtenido para cada caso.

\begin{tabular}{ccc}
\hline Catálogo & $\gamma[$ adim $]$ & {$\left[\boldsymbol{R}^{2}\right]$} \\
\hline Gardner & $0.1781 \pm 0.009$ & {$[0.97]$} \\
Clapp-Horn. & $0.3145 \pm 0.023$ & {$[0.94]$} \\
vG-M. & $0.2596 \pm 0.021$ & {$[0.89]$} \\
\hline
\end{tabular}
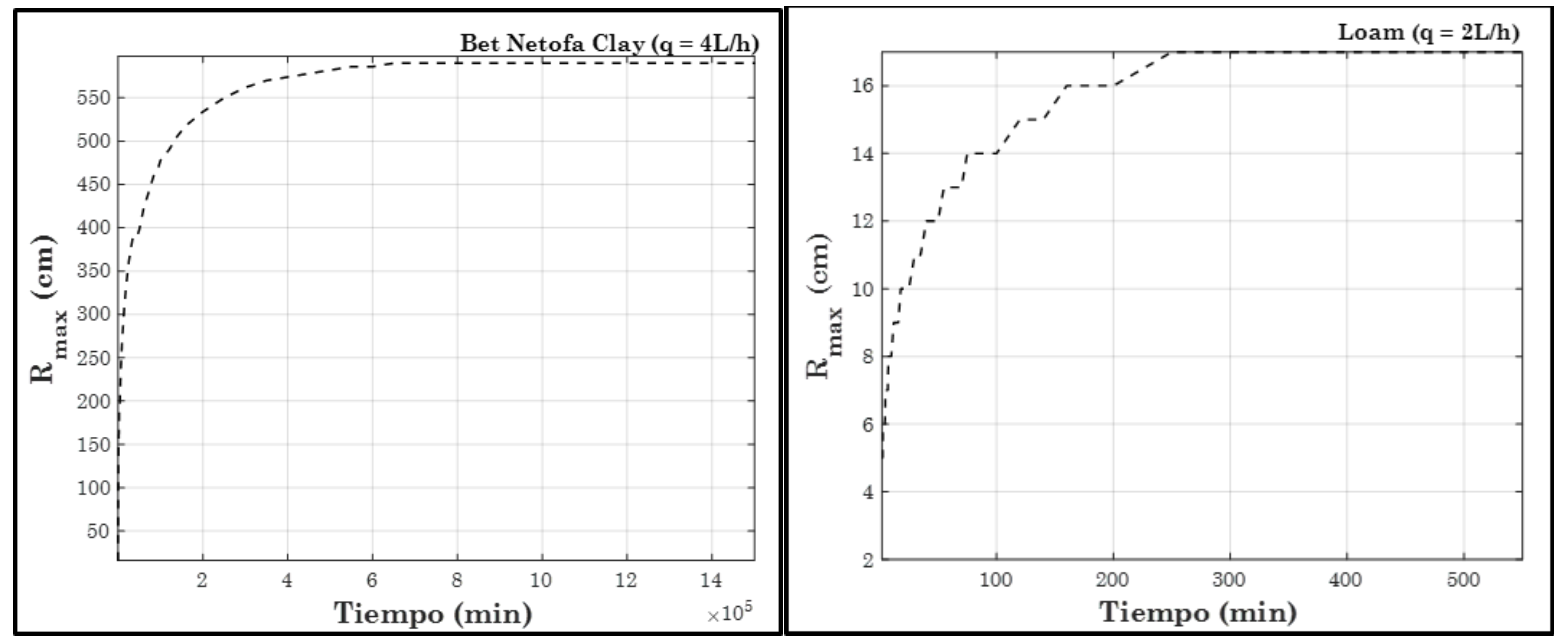

Figura 2. Ejemplos de simulación. Evolución del radio máximo en función del tiempo para dos suelos de los catálogos de Mualem (a) y Clapp-Hornberger (b). Se ha considerado que se alcanza el radio máximo (régimen estacionario) cuando este parámetro permanece constante durante al menos el 30\% del tiempo total de simulación.

\subsection{Discusión del modelo empírico.}

De las simulaciones realizadas se han extraido algunas conclusiones generales con respecto al radio de la zona saturada y que son comunes a los tres catálogos: (a) el radio del charco avanza más deprisa, para los suelos con mayor carácter arcilloso que son aquellos en los que el producto $k_{s} \Delta \theta$ es más pequeño, (b) el radio límite es mayor y se alcanza más tarde para los suelos de carácter arcilloso, es decir, cuando $k_{s} \Delta \theta$ es más pequeño, (c) el contenido de humedad inicial afecta a la evolución de la zona saturada de modo que cuanto mayor es el contenido de humedad inicial más rápido y más grande crece el charco (zona saturada), (d) la representación del radio límite para todos los tipos de suelo de un mismo catálogo sigue la tendencia $\left(k_{s} \Delta \theta\right)^{\delta}$ donde $\delta$ es en este caso un parámetro constante para 


\section{Congreso Nacional de Riegos CARTAGENA 2021}

cada catálogo, y muy próximo $-1 / 2$ independiente del caudal aplicado y (e) se ha comprobado que el caudal aplicado afecta al radio máximo como una función raíz cuadrada para los suelos de los tres catálogos. Por todo ello, se ha concluido que el modelo empírico que mejor ajusta al radio máximo del charco en función de estos parámetros el siguiente (ajuste de la Figura 1):

$$
R_{0}=\gamma \sqrt{\frac{q}{k_{s} \Delta \theta}}
$$

Siendo esta una ecuación común para los suelos caracterizados con los tres tipos de funciones y catálogos mencionados. Los valores obtenidos para el factor adimensional $\gamma$ se han presentado en la Tabla 1. Observar que el ajuste es satisfactorio a pesar de que la constante de capilaridad macroscópica $\alpha$, que está relacionada con el tamaño del poro, no aparece en el modelo. Este hecho representa que el efecto de este parámetro sobre el radio límite es pequeño; además, al estar relacionado el tamaño del poro con la diferencia de contenidos de humedad $\Delta \theta$, se puede argumentar que la información que representa $\alpha$ ya está incluida en $\Delta \theta$.

Para los suelos del tipo Gardner se ha comprobado que en la mayoría de los casos el radio obtenido es similar al que predice el modelo de Wooding, o sensiblemente superior. La desviación entre los dos modelos es en promedio inferior al $9 \%$, siendo de tan solo un $1.7 \%$ para los casos en los que el radio límite supera $10 \mathrm{~cm}$. Esto significa que el ajuste entre los dos modelos es mejor para suelos francos y arcillosos donde el tamaño del charco en régimen estacionario es mayor. Con suelos arenosos la aproximación analítica de Wooding subestima el radio límite obtenido en las simulaciones. Este hecho confirma los resultados reportados por Pullan y Collins, Weir y Warrick [9-11], que también han mencionado un pobre ajuste de la expresión de Wooding con respecto a soluciones numéricas propias bajo condiciones de radios límite pequeño. No obstante, también hay que tener en cuenta los errores de las soluciones numéricas para tiempos largos de simulación.

\subsection{Comparación del modelo empírico y analítico.}

La expresión analítica obtenida para un disco superficial de radio saturado y constante en el límite en que el tiempo tiende a infinito, ecuación (2b-REF.1), es equivalente al régimen estacionario que representa el modelo empírico (3) si se considera despreciable el primer término del factor $c_{g}$. Esto equivale a decir que el efecto de la succión es depreciable frente al gravitatorio, lo que es asumible desde un punto de vista físico en este contexto:

$$
R_{0}=\frac{1}{2^{3 / 4} \pi^{1 / 2}} \sqrt{\frac{q}{\Delta \theta k_{s}}}
$$

Lo que se corresponde a un valor para la constante $\gamma=0.3355$. Esta simplificación es asumible ya que para tiempos largos el efecto predominante en el flujo es el gravitatorio. Advertir que esta ecuación es general para cualquier tipo de suelo que pueda ser definido a través de los tres parámetros propios de las expresiones analíticas propuestas en el modelo analítico [19]. Notar que despreciando la succión, no hay dependencia entre el radio máximo que describe el modelo analítico y la tensión en el frente de 


\section{Congreso Nacional de Riegos CARTAGENA 2021}

avance que esta asociada a este modelo. Advertir que, según el modelo analítico propuesto tampoco existe dependencia entre el radio límite del régimen estacionario y el parámetro $\alpha$.

Utilizando todos los términos de la ecuación (2b-REF.1), y por tanto, asumiendo el efecto de la succión propia del suelo aparece el término de la tensión en el frente de avance $\left(\tau_{f}\right)$ en la ecuación del radio límite:

$$
R_{0}=\frac{\tau_{f}}{4 \Delta \theta}\left[\sqrt{1+\frac{8 \Delta \theta q}{\sqrt{2} \pi k_{s} \tau_{f}^{2}}}-1\right]
$$

Donde se ha omitido la solución negativa propia de esta ecuación de segundo grado. La Figura 3 compara las dos últimas expresiones sobre variables adimensionales para el radio y caudal:

$$
\begin{gathered}
R_{0}^{*}=\sqrt{q^{*}} \quad(4 b) \\
R_{0}^{*}=\sqrt{1+q^{*}}-1 \quad(5 b) \\
R_{\text {ref }}=\frac{\tau_{f}}{4 \Delta \theta} \quad ; \quad q_{\text {ref }}=\frac{\sqrt{2} \pi k_{s} \tau_{f}^{2}}{8 \Delta \theta} \quad \text { [REF.2] }
\end{gathered}
$$

(a)

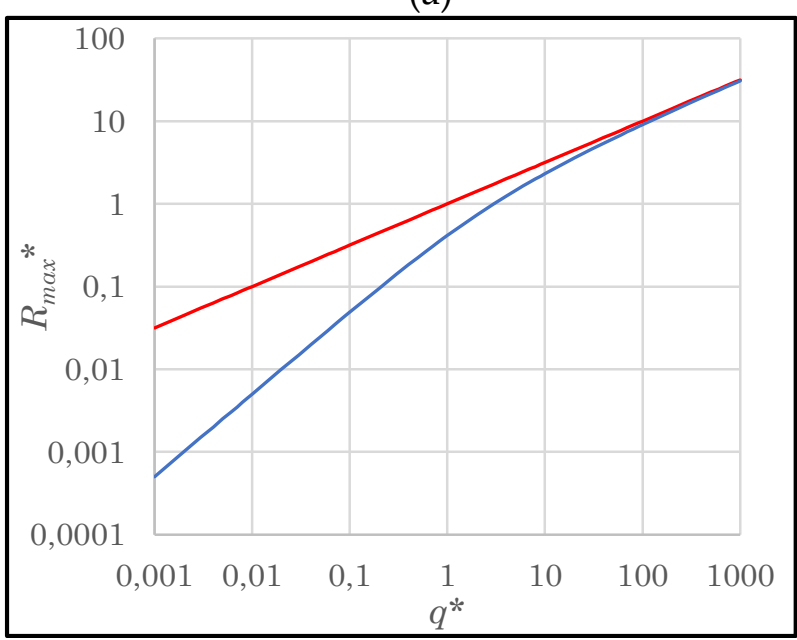

(b)

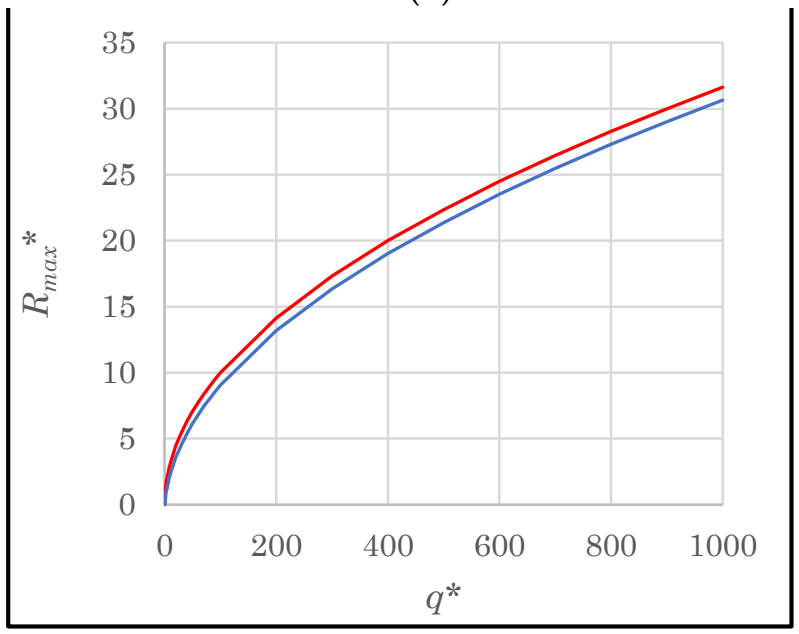

Figura 3. Comparación de las ecuaciones adimensionales (4b) en color rojo y (5b) línea azul. (a) Escala logarítmica y (b) escala decimal.

Se puede observar como el radio que representa la línea roja (4b) es mayor para todos los valores del caudal que el de la línea azul, ecuación (5b). Obviamente, al considerar el efecto de la succión (por pequeño que sea) la filtración es mayor, y por tanto el radio límite en régimen estacionario es mas pequeño. Las diferencias en términos absolutos son mayores para caudales altos, aunque no superan el 3.2\% dentro del rango de estudio. Las diferencias relativas son significativas para valores pequeños del caudal (donde el efecto de la succión es más importante) como se puede observar en el diagrama logarítmico, aunque desaparecen para valores intermedios del caudal. 


\section{Conclusiones}

Las simulaciones realizadas conducen a la conclusión de que el radio máximo de la zona saturada en superficie depende principalmente del caudal aplicado, la conductividad hidráulica en saturación, y la diferencia de los contenidos de humedad inicial y en saturación del suelo. La independencia de la expresión empírica inferida del parámetro $\alpha$ (constante de escala macroscópica de capilaridad) supone una ventaja con respecto a otros modelos de referencia como el modelo analítico de Wooding, ya que este parámetro es de obtención experimental más compleja; además, el hecho de que la solución analítica propuesta tampoco dependa de $\alpha$ justifica la validez del modelo empírico en este aspecto.

Por otro lado, es necesario recordar que la solución analítica de Wooding fue derivada para suelos caracterizados según las funciones del tipo Gardner, por lo hay que asumir que tan solo es válida en este contexto. En este sentido, se realizó un grupo de simulaciones para tres tipos de suelo caracterizado con funciones del tipo van Genuchten-Mualem en las que se encontró una desviación promedio del $48 \%$ entre el radio máximo simulado y el radio que predice la aproximación de Wooding, el cual, estaba subestimado. Recordar que según se ha reportado en [9-11] para suelos del tipo Gardner la solución de Wooding también tiende a subestimar sensiblemente su predicción del radio límite para caudales pequeños y suelos arenosos donde el tamaño del radio máximo en régimen estacionario es menor a 10 $\mathrm{cm}$. Un comportamiento similar al descrito por estos autores se ha encontrado en las simulaciones realizadas. Por otro lado, las expresiones inferidas (3) para los suelos este tipo (vG-M), generan valores para el radio máximo que no siguen ningún patrón sistemático de desviación respecto a las simulaciones realizadas de comprobación, y que según se ha visto, son en promedio inferiores al 15\%.

Observar que la desviación entre la ecuación analítica simplificada respecto al modelo empírico inferido es del 6\% para el caso de suelos del tipo Clapp y Hornberger, y del 22\% para los suelos caracterizados por funciones van Genuchten-Mualem. Es necesario recordar que la expresión analítica propuesta no sigue un patrón de caracterización del suelo similar al de estos dos modelos. Se concluye por tanto que, los dos modelos analítico (4a) y empírico (3) propuestos, pueden ser una alternativa de interés para el desarrollo y diseño de los sistemas de riego, y en particular, el posicionamiento de goteros, en los casos en que, como por ejemplo [22], se utilice el parámetro del radio máximo del charco en función del caudal aplicado para este efecto. 


\section{Congreso Nacional de Riegos CARTAGENA 2021}

\section{Referencias}

1. Schwartzman M.; Zur B. Emitter Spacing and Geometry of Wetted Soil Volume. J. Irrig. Drain. Eng. 1986. 112(3): $242-253$

2. Amin M. S. M.; Ekhmaj A. I. M. DIPAC-DripIrrigation Water Distribution Pattern Calculator. 7th International Micro-Irrigation Congress, 10-16 Sept. Pwtc, Kuala Lumpur, Malaysia. 2006.

3. Keyvan M.; Peters R.T. Wetting patterns models for drip irrigation: new empirical model. Journal of Irrigation and Drainage Engineering. 2011. 137(8): 530-36.

4. Kaul R.K.; Michael A.M. Moisture front advance under point source of water application. J Agric Eng. 1982. 19(2):1-8

5. Subbaiah R.; Pandey R.N. Soil moisture movement under trickle irrigation. J Inst Eng (India). 1991. 18:22-28

6. Zazueta F.S.; Clark G.A.; Smajstrla A.G.; Carrillo M. A simple equation to estimate soil-water movement from a drip irrigation source. Proceedings of 5 th international microirrigation congress on conserving resource/preserving the environment, ASABE, pp 581-856. 1995.

7. Wooding R.A. Steady infiltration from a shallow circular pond. Water Resour Res. 1968. 4:1259-73.

8. Gardner W.R. Some steady state solutions of unsaturated moisture flow equations with application to evaporation from a water table. Soil Sci. 1958. 85:228-232.

9. Pullan A.J. y Collins I.F. Two and three-dimensional steady quasilinear infiltration from buried and surface cavities using boundary element techniques. Water Resour Res. 1987. 23(8):1633-1644.

10. Weir G.J. Steady infiltration from small shallow circular ponds. Water Resour Res. 1987. 23:733-736.

11. Warrick A.W. Models for disk infiltrometer. Water Resour Res. 1992. 28:1319-1327.

12. del Vigo Á., Zubelzu S. \& Juana L. Numerical routine for soil dynamics from trickle irrigation. Applied Math. Model. 2020. 83: 371-385.

13. del Vigo Á., Zubelzu S. \& Juana L. Algoritmo para la resolución de la ecuación de Richards en 3-D para riego por goteo: Método, validación y resultados preliminares. XXXVII Congreso Nacional de Riegos. Don Benito. Spain. 2019a.

14. del Vigo Á., Zubelzu S. \& Juana L. Study of water infiltration in soil by Richards equations in 3D: summary and methodology validation. 11th World Congress on Water Resources and Environment. Madrid. 2019b.

15. del Vigo, Á. Simulación del flujo del agua en el suelo en riego por goteo superficial, soluciones analíticas aproximadas, caracterización del suelo y diseño de los riegos. Phd. Thesis, Universidad Politécnica de Madrid. Madrid. 2020.

16. Clapp R.B. y Hornberger G.M. Empirical equations for soil hydraulic properties. Water Resour Res. 1978. 14:601-604.

17. van Genuchten M.Th. A closed-form equation for predicting the hydraulic conductivity of unsaturated soils. Soil Sci Soc Am Proc. 1980. 44:892-898

18. del Vigo Á., Zubelzu S. \& Juana L. Soluciones analíticas aproximadas bajo hipótesis de Green-Ampt desde fuentes semiesférica y circular en superficie. Jornadas de Ingeniería del agua. Toledo. 2019c.

19. del Vigo Á., Zubelzu S. \& Juana L. Infiltration models and soil characterization for hemispherical and disc sources based on Green-Ampt assumptions. Journal of Hydrology. 2021. 595: 1259-66.

20. Šimůnek J., van Genuchten M. \& Šejna M. The HYDRUS Software Package for Simulating the Two- and Three-Dimensional Movement of Water, Heat, and Multiple Solutes in Variably-Saturated Media, Technical Manual Version 1.0. University of California Riverside. Riverside, CA, 3PC. Progress, Prague. Czech Republic. 2006.

21. Mualem, Y. A new model for predicting the hydraulic conductivity of unsaturated porous media. Water Res Jour. 1976. 12: 513.

22. Bresler, E. Analysis of trickle irrigation with application to design problems. Irrigation Science. 1978. 1: 3-17. 